9. Bahcall, J. N. \& Woltjer, L. Nature 274, 22-23 (1974).

10. Burbidge, E. M., Burbidge, G. R. \& O'Dell, S. L. Natur 248, 568-569 (1974)

11. Gott, J. R. III \& Gunn, J. E. Astrophys. J. 190, L105-L108 (1974).

12. Bolton, J. G., Peterson, B. A., Wills, B. J. \& Wills, D. Astrophys. J. 210, L1-L3 (1976)

13. Wills, D. Phys. Scr. 17, 333-337 (1978)

14. Shaver, P. A. Astr. Astrophys. 136, L9-L10 (1984).

15. Owen, F. N., Wills, B. J. \& Wills, D. Astrophys. J. 235 L57-L60 (1980)

16. Harris, D. E., Dewdney, P. E., Costain, C. H., Butcher, H. \& Willis, A. G. Astrophys. J. 270, 39-47 (1983).

BURBIDGE ET AL. REPLY-In answer to Shaver's comments we wish to make the following points:

(1) We did not reference most of the work cited by him (his refs 2-5) because it refers to pairs of much wider angular separation than ours. We wish to emphasize again that it is the pairs with very small separations that should be looked for as indicators of physical associations.

(2) While we agree that Shaver's work is not consistent with ours, the results given in Burbidge et al. ${ }^{1}$ are to be compared with the prediction of the numbers expected by chance made in 1974 by Burbidge et $a l^{2}$.

(3) This comment by Shaver and earlier work by him $^{3}$ and by Wills ${ }^{4}$ describes results consistent with the non-clustering of QSOs. However, the proponents of the cosmological hypothesis must have cause to worry about results like ours which appear to be inconsistent with that hypothesis. Shaver's earlier work and the present paper do not explain the discrepancy.

G. R. BURBIDGE

J. V. NARLIKAR

A. HEWITT

Center for Astrophysics and

Space Sciences,

University of California, San Diego,

La Jolla, California 92093, USA

Taba Institute of Fundamental Research, Bombay, India

1. Busbidge, G. R., Narlikar, J. V. \& Hewitt, A. Nature 317, 413-415 (1985)

2. Burbidge, E. M., Burbidge, G. R. \& O'Dell, S. L. Nature 248, 568-569 (1974)

3. Shaver, P. A. Astr. Astrophys. 143, 451-454 (1985).

4. Wills, D. Phys. Sci. 17, 333-337 (1978).

\section{Homology between IgE-binding factor gene and endogenous retroviruses}

RECENTLY, Toh et $a l^{1}$, reported the detection of nucleotide sequence homology between segments of a rodent IgE-binding factor (IgE-BF) gene ${ }^{2}$ and a Syrian hamster intracisternal A particle (IAP) genome ${ }^{3}$. Toh et al. proposed that the IgE-BF gene is a hybrid gene which evolved very recently by integrating segments of genes of retroviral origin with a cellular gene coding for an IgE-BF domain ${ }^{1}$. Our own recent studies ${ }^{4}$ have shown that the IgE-BF cDNA is, in fact, derived from a member of the retroviruslike mouse IAP sequence family, with its

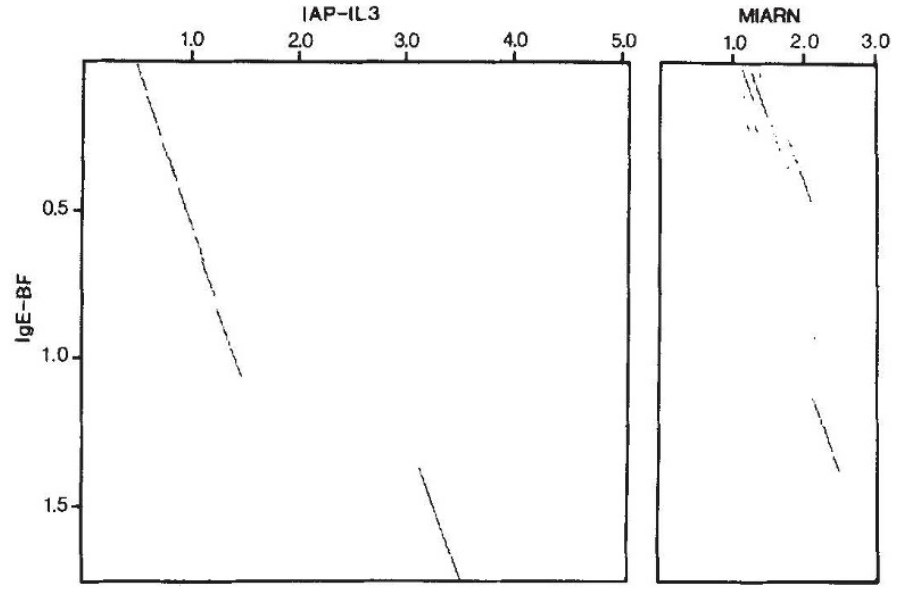

Fig. 1 Diagonal plots showing the nucleotide sequence homology between the $\lg$ E-BF cod ing sequence ${ }^{2}$ and the murine IAP genomes IAP-IL3 (ref. 4) and MIARN (ref. 6). The comparisons were made using the program DIAGON ${ }^{10}$ with span length $=21, \%=16$. The dots indicate segments of homology of $76 \%$ or greater. The scale for each of the sequences is in kilobases.

coding sequence totally derived from mouse IAP gag and pol genes.

We recently sequenced a mouse IAP genome (IAP-IL3) found in the rearranged interleukin-3 gene of the mouse myelomonocytic leukaemia cell line WEHI-3B (ref. 5). Comparison of the coding region of the IgE-BF gene with the 5.1-kb IAP-IL3 sequence ${ }^{4}$ showed a very high level of homology extending across the majority of the IgE-BF coding region in two major segments (Fig. 1). Most of the region of the IgE-BF coding sequence which is missing from IAP-IL3 is found in another mouse IAP element, MIARN (ref. 6) (Fig. 1). Thus, overall, 97\% of the IgE-BF coding sequence is present in the two endogenous mouse IAP elements at an average homology of $90 \%$. The restriction map of the noncoding region of the IgE-BF cDNA is very similar to that of the terminal region of IAP-IL3 suggesting that the homology extends to this region also.

The IgE-BF cDNA clone was isolated from the rat-mouse $T$-cell hybridoma 23B6 prepared by fusing rat $T$ lymphocytes with cells of the AKR mouse thymoma BW5147 (ref. 7). Although one would have expected to isolate cDNA clones for rat IgE-BF from this cell line, the very high level of sequence homology with mouse IAP elements indicates that the IgE-BF cDNA was derived from a mouse IAP genome of the fusion partner. Hybridization analysis using mouse IAP gene probes has shown that mouse and rat IAP elements do not share the very high levels of homology observed between the $\mathrm{IgE}$ $\mathrm{BF}$ gene and the mouse IAP elements ${ }^{8}$. The size of the cDNA clone $(\sim 3.3 \mathrm{~kb})$, together with the restriction map of its $3^{\prime}$ noncoding region suggests it was derived from a genomic RNA of a deleted IAP provirus which is intermediate in size between IAP-IL3 and MIARN. This conclusion is also in agreement with the recent findings of Moore et al. ${ }^{9}$.

The significance of this relationship between the IgE-BF gene and mouse IAP elements is difficult to assess without further work, including the determination of whether a similar relationship also exists between IgE-BF genes and IAP-like elements in other mammals. It does, however, raise the interesting possibility that some members of the highly reiterated mouse IAP sequence family may have evolved to encode proteins with biological functions unrelated to retroviral replication.

SANIE YMER

IAN G. YOUNG

Medical Molecular Biology Unit,

John Curtin School of Medical Research, Australian National University, Canberra, A.C.T., 2601 Australia

1. Toh, H., Ono, M. \& Miyata, T. Nature 318, 388-389 (1985) 2. Martens, C. L. et al. Proc, natn. Acad. Sci. U.S.A. 82 2460-2464 (1985)

3. Ono, M., Toh, H., Miyata, T. \& Awaya, T. J. Virol. 55, 387-394 (1985).

4. Ymer, S., Tucker, W. Q. J., Campbell, H. D. \& Young, I. G. Nucleic Acids Res. 14, 5901-5918 (1986).

5. Ymer, S., Tucker, W. Q. J., Sanderson, C. J., Hapel, A. J., Campbell, H. D. \& Young, I. G. Nature 317, 255-258 (1985)

6. Burt, D. W., Reith, A. D. \& Brammar, W. J. Nucleic Acids Res. 12, 8579-8593 (1984).

7. Huff, T. F, Uede, T. \& Ishizaka, K. J. Immun. 129, 509-514 (1982)

8. Lueders, K. K. \& Kuff, E. L. Nucleic Acids Res. 9, 5917-5930 (1981).

9. Moore, K. W et al J. Immun. 136, 4283-4290 (1986). 10. Staden, R. Nucleic Acids Res. 10, 2951-2961 (1982)

\section{Matters Arising}

Matters Arising is meant as a vehicle for comment and discussion about papers that appear in Nature. The originator of a Matters Arising contribution should initially send his manuscript to the author of the original paper and both parties should, wherever possible, agree on what is to be submitted. Neither contribution nor reply (if one is necessary) should be longer than 500 words and the briefest of replies, to the effect that a point is taken, should be considered. 